\title{
Consecutive successful pregnancies subsequent to intravenous immunoglobulin therapy in a patient with recurrent spontaneous miscarriage
}

This article was published in the following Dove Press journal:

International Medical Case Reports Journal

II December 2015

Number of times this article has been viewed

\author{
Michael F Diejomaoh ${ }^{1,2}$ \\ Zainab Bello² \\ Waleed Al Jassar ${ }^{1,2}$ \\ Jiri Jirous ${ }^{2}$ \\ Kavitha Karunakaran ${ }^{2}$ \\ Asiya T Mohammed' \\ 'Department of Obstetrics and \\ Gynaecology, Faculty of Medicine, \\ Kuwait University, Safat, ${ }^{2}$ Maternity \\ Hospital, Shuwaikh, Kuwait
}

\begin{abstract}
Background: Recurrent spontaneous miscarriage (RSM) has a multifactorial etiology, mainly due to karyotype abnormalities including balanced translocation, anatomical uterine disorders, and immunological factors, although in $50 \%-60 \%$ the etiology is unexplained. The treatment of RSM remains challenging, and the role of intravenous immunoglobulin (IVIG) in RSM is controversial.

Case report: Mrs HM, 37 years old, obstetric summary: P0+1+13+1, a known case of hypothyroidism/polycystic ovary syndrome, married to an unrelated 47-year-old man, presented to our RSM clinic in early January 2014 for investigation and treatment. She has had multiple failed in vitro fertilization trials and 13 first-trimester missed miscarriages terminating at 6-7 weeks, all without IVIG therapy. Her tenth pregnancy was spontaneous, managed in London, UK, with multiple supportive therapy and courses of IVIG starting from the third to the 30th week of pregnancy. The pregnancy ended at 36 weeks of gestation with a cesarean section and a live girl baby was delivered. Mrs HM had balanced translocation, 46XX t (7:11) (p10:q10). Preimplantation genetic diagnosis/intracytoplasmic sperm injection/in vitro fertilization was performed with embryo transfer on May 29, 2014, and resulted in a successful pregnancy. She was commenced immediately on metformin, luteal support, and IVIG therapy, started at 6 weeks of gestation and at monthly intervals until 30 weeks of gestation, and also received additional therapy. The pregnancy was monitored with ultrasound, progressed uneventfully until admission at 35 weeks of gestation, with mildly elevated liver enzymes and suspected fetal growth restriction. She was managed conservatively, and in the light of nonreassuring fetal status, a live female infant weighing $2.29 \mathrm{~kg}$ was delivered by emergency cesarean section on January 14, 2015, with an Apgar score of 8 and 9 and mild respiratory distress, and was admitted to the Special Care Baby Unit for intensive therapy. The mother and baby made satisfactory progress and were discharged on January 24, 2015.
\end{abstract}

Conclusion: Two consecutive successful pregnancies in Mrs HM with multiple causes of RSM treated with other medications and IVIG strongly suggest that IVIG has a positive role in RSM.

Keywords: recurrent, miscarriage, intravenous immunoglobulin, pregnancy outcome

\section{Introduction}

Miscarriage is the most frequently encountered complication of pregnancy, ${ }^{1-3}$ and miscarriage rates of $10 \%-30 \%$ have been reported by various authors. ${ }^{4,5}$ Spontaneous miscarriage presents in diverse ways and is subclassified into distinct subtypes. In this report, recurrent spontaneous miscarriage (RSM) is defined as the loss of three or more consecutive pregnancies before 20 weeks of pregnancy, and it is reported to occur in $0.5 \%-1 \%$ of women in reproductive age.$^{1,3,6-8}$ Ectopic pregnancies and molar pregnancies are not included in this definition. There is a lack of uniformity in the definition of RSM. ${ }^{9}$
Correspondence: Michael F Diejomaoh Department of Obstetrics and Gynaecology, Faculty of Medicine, Kuwait University, PO Box 24923.

Safat I3II0, Kuwait

Tel +965 253I 960I/+965 66741876

Fax +96525338906

Email michaeldiejo@hotmail.com 
Many authors define RSM as the loss of two or more consecutive pregnancies, ${ }^{10-12}$ and the incidence may increase to $5 \%$. This definition is particularly applicable to women older than 35 years. In Kuwait, we commence investigations in women aged 35 years and older who have had two or more consecutive miscarriages. ${ }^{9}$ RSM may be primary, secondary, or rarely tertiary. Tertiary RSM refers to the event that follows secondary RSM. A patient who has had a series of miscarriages without a previous viable pregnancy or birth (live birth or stillbirth) is classified as primary RSM, whereas a patient who has had a series of miscarriages after a viable pregnancy, a live birth, or a stillbirth is classified as secondary RSM. ${ }^{13}$

The etiology of RSM may be multifactorial, and the more established etiological factors include antiphospholipid syndrome, anatomical uterine structural anomalies, and parental

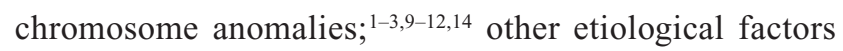
include endocrine disorders and inherited thrombophilia. However, in $40 \%-50 \%$ of the cases of RSM, the etiology cannot be identified or established, and the cause is then defined as idiopathic or unexplained. ${ }^{9,11,12}$ Immunological disturbances and aberrations seem to be the risk factors in many cases of RSM and have been suggested to play an important role in the etiology of RSM. ${ }^{7,15-18}$ Elevated natural killer (NK) cells $\left(\mathrm{CD} 56^{+} / \mathrm{CD} 16^{+}\right)$have been implicated in the etiology of RSM through their interaction in the maternal/ fetal interface, ${ }^{16,19,20}$ and the higher levels of NK cells in the nonpregnant women have been associated with the increased probability of miscarriage in a subsequent pregnancy. ${ }^{21,22}$

The establishment of the cause of RSM in any patient requires detailed and comprehensive investigations. 4,9,11,12,14 The etiology of RSM may be multifactorial in some patients and therefore calls for extensive elaborate investigations. The details of such investigations of RSM will not be covered in this report.

The treatment of RSM remains varied and challenging, and where the cause is known, such as in antiphospholipid syndrome, the treatment could be easily executed. However, in many cases of RSM, especially the idiopathic cases of RSM, which constitute $40 \%-50 \%$ of cases of RSM, the treatment may be empirical and not evidence based. ${ }^{9,23}$ Patients and their treating doctors may therefore face challenges and frustrations from the treatment of RSM because of the uncertain efficacy of the treatment being offered. ${ }^{24}$

Intravenous immunoglobulin (IVIG) therapy has been administered to many patients with RSM on a trial basis in double-blind, placebo-controlled trials with the understanding that there may be an immunological basis for the pathogenesis of such cases of RSM. ${ }^{1-7,25-27}$ IVIG has also been reported to enhance live births in patients with RSM who have been demonstrated to have elevated NK cells. ${ }^{20,23,28}$

However, the role of IVIG therapy in RSM, which has been the subject of multiple trials, meta-analyses, and systemic reviews, ${ }^{1-3,15,29}$ has remained controversial, and the results of such trials have been varied with contrasting results and deductions. IVIG therapy resulted in positive outcome in idiopathic secondary RSM, but no benefits were observed for primary RSM; $58 \%$ of patients with secondary RSM had successful pregnancies after IVIG therapy compared with that of $24 \%$ of placebo-treated patients. ${ }^{7}$ Positive pregnancy outcome was also reported in patients with secondary RSM treated with IVIG therapy in randomized trials. ${ }^{1,8,25-27}$ However, the meta-analysis of eight randomized controlled trials concluded that there was a positive pregnancy outcome in women with secondary RSM treated with $\mathrm{IVIG}^{29}$ and other meta-analyses and systemic reviews reported no benefits for IVIG therapy in RSM. ${ }^{1-3,18}$ In many studies of IVIG therapy in RSM quoted earlier, the dosage regimes of IVIG used and the treatment protocol followed were diverse and varied. These may have contributed to the diverse results reported in these studies.

As highlighted previously, the etiology of RSM may be multifactorial, and the multiple causes of RSM may be present in the same patient. It is also pertinent to state that as the number of miscarriages increases in the same patient, as the patient therefore increases in age, the chances of a successful pregnancy outcome in a subsequent pregnancy will diminish and the stress and anxiety will increase in such patients, and the challenge to the treating physician and patient becomes phenomenal.

A major area of challenge is in the area of parental chromosome aberrations/anomalies (including balanced translocations and chromosome inversions) where technological approaches to diagnosis and management are necessary. In the presence of a known parental balanced translocation as the cause of RSM, preimplantation genetic diagnosis (PGD) with in vitro fertilization (IVF) is an accepted modality of treatment. ${ }^{9,12}$ The chances of these procedures leading to a successful pregnancy are not always guaranteed, and thus, a successful pregnancy may not always be achieved.

We present a case of high-order RSM (many previous miscarriages) due to multiple etiological factors that are fully investigated and treated successfully with PGD/intracytoplasmic sperm injection (ICSI)/IVF, multiple therapy, and IVIG, which resulted in a successful pregnancy outcome.

\section{Case report}

Mrs HM, 37 years old, P0+1+13+1, a known case of hypothyroidism due to autoimmune thyroiditis and polycystic 
ovary syndrome (PCOS), married to an unrelated 47-year-old civil servant (nonconsanguineous marriage), presented to our RSM clinic at maternity hospital, Kuwait, in early January 2014, for further investigations and treatment. Written consent was obtained from the patient before commencement of the IVIG therapy. Verbal consent was obtained from the patient before the case report was prepared. Ethical approval was not necessary for this case report. All principles outlined in the Declaration of Helsinki were followed. The patient had been married to the same husband for 27 years. A comprehensive medical/surgical history was obtained and investigations commenced. Her surgical history revealed that she had wedge resection of the ovaries in 2002 for PCOS and her menstrual cycles were reported as regular.

A summary of her obstetric history revealed that she has had nine first-trimester miscarriages that terminated at 6-7 weeks of gestation. No IVIG therapy was offered during these pregnancies. Evacuation of the uterus was performed in six of these miscarriages, and there were no complications. She has had six failed IVF trials.

Her tenth pregnancy was spontaneous and was managed in London, UK, where she had also been diagnosed with hypothyroidism and elevated NK cells, from late 2009 to early 2010. She received multiple doses of IVIG starting from the third week of pregnancy until 30 weeks of gestation and other forms of multiple supportive therapy, which included Eltroxin and Glucophage (metformin) tablets, enoxaparin (Clexane) injections, low-dose aspirin tablets, and progesterone tablets. This pregnancy ended at 36 weeks of gestation with a cesarean section because of the failure to progress in labor and a healthy live girl baby was delivered in February 2010.

She had another four consecutive first-trimester missed miscarriages; none of these pregnancies were treated with IVIG. Her last spontaneous pregnancy was in November 2010 , and this was followed by a period of 3 years of secondary infertility, which was investigated.

During her first visit to the RSM clinic at maternity hospital, Kuwait, in early January 2014, a full physical examination revealed no abnormalities apart from a high body mass index of $32 \mathrm{~kg} / \mathrm{m}^{2}$, and she was fully investigated again for the RSM. Full immunological, endocrinological, ultrasound, and other blood investigations (including anticardiolipin antibodies [IgG/IgM], lupus anticoagulant, thrombophilia screening, thyroid function tests, serum prolactin, 21 st day serum progesterone, and 3D ultrasound studies) were performed, and they revealed no abnormalities.

Karyotype studies revealed that her husband was 46XY and the patient had a balanced translocation - 46XX t (7:11) (p10: q10). Further investigations revealed that the patient had elevated NK cells $\left(\mathrm{CD} 56^{+} / \mathrm{CD}^{+} 6^{+}\right)$, and oligoasthenozoospermia was detected in her husband. Mrs HM had been commenced on $75 \mu \mathrm{g}$ Eltroxin daily and $850 \mathrm{mg}$ metformin tablets twice daily accordingly as part of the treatment for her previously diagnosed hypothyroidism and PCOS and was advised to undergo PGD/ICSI/IVF.

The diagnoses of Mrs HM at this point were previous cesarean section, RSM due to multiple etiological factors of chromosome anomaly, elevated NK cells, hypothyroidism, and PCOS. Her age, 37 years, and moderate obesity were additional contributory factors.

The procedures (PGD/ICSI/IVF) recommended for Mrs HM were successfully performed, and the embryo transfer was performed on May 29, 2014. A successful pregnancy was confirmed, and this was considered as a very precious and high-risk pregnancy. Routine antenatal blood tests and ultrasound revealed normal results. The patient was commenced on $5 \mathrm{mg}$ folic acid tablets, metformin tablets, vitamins, low-dose aspirin, Clexane $40 \mathrm{mg}$ subcutaneous injections, and $75 \mu \mathrm{g}$ Eltroxin daily. After appropriate consent, IVIG (Biotest, Germany) therapy was commenced at 6 weeks of gestation after positive fetal cardiac activity had been confirmed. Luteal support was also commenced with progesterones (Cyclogest pessaries) $400 \mathrm{mg}$ twice daily vaginally and oral dydrogesterone (Duphaston) tablets $10 \mathrm{mg}$ twice daily.

Luteal support with progesterone suppositories and metformin tablets was continued until the 13 th week of pregnancy. Cervical cerclage was performed at 14 weeks of gestation by a colleague in private practice. IVIG $(500 \mathrm{mg} / \mathrm{kg}$, in divided doses, intravenous for 2 days every month) was continued after the first dose at 6 weeks of gestation. The patient was always admitted to the hospital for the IVIG therapy, closely monitored for any adverse/untoward reactions, and discharged home after each treatment cycle. Serial ultrasound examinations were performed, and at 20 weeks of gestation, the scan revealed no fetal anomalies. Dydrogesterone treatment was continued until 24 weeks of gestation while all the other drugs listed earlier were continued. At approximately 16 weeks of gestation, folic acid therapy was stopped, and supplemental folic acid and oral iron (single tablet) were continued until term. In view of the history of previous preterm delivery, injection primolut depot (17 $\alpha$-hydroxy progesterone caproate) $500 \mathrm{mg}$ by the intramuscular route weekly was commenced after 16 weeks of gestation and was continued until term (37 weeks of gestation). She was also screened for diabetes mellitus with oral glucose tolerance test that revealed normal results. 
The pregnancy progressed uneventfully, and the growth of the baby was satisfactory. The monthly IVIG therapy was stopped at 30 weeks of gestation after due counseling and discussion with the patient; she had received a total of seven doses of IVIG injections with no adverse fetal or maternal effects being reported. During her previous successful pregnancy, IVIG was continued until 30 weeks of gestation and the same has been followed in the current pregnancy also.

At 33 weeks of gestation, on December 28, 2014, she presented with slight painless vaginal bleeding and was admitted to the labor ward of maternity hospital with the diagnosis of antepartum hemorrhage (APH). Ultrasound examination confirmed the diagnosis of APH due to placenta previa marginalis (the placenta was situated posteriorly, $1.2 \mathrm{~cm}$ from the cervical os). Routine blood investigations revealed normal results. She was managed conservatively along departmental guidelines (these were identical to the Royal College of Obstetricians and Gynaecologists [RCOG]/ American College of Obstetricians and Gynecologists [ACOG]/ Society of Gynecologists of Canada [SOGC] guidelines). Dexamethasone (12 mg, 12 hourly for 24 hours by intramuscular injections) was administered to the patient. Blood was crossmatched (in case there was need for immediate delivery), and a high-risk consent was obtained from the patient and her husband. The conservative treatment was to be continued until 37 weeks of gestation when an elective cesarean section was planned. After stabilization of the patient in the labor ward and a stay of 14 hours, she was transferred to the antenatal ward.

The patient presented with generalized pruritus on January 10, 2015, and there was neither nausea/vomiting nor jaundice. A diagnosis of cholestasis of pregnancy was made, and the patient was jointly managed conservatively in collaboration with the physician. The liver enzymes were slightly elevated (alanine aminotransferase [ALT], 77 IU/L [normal range, 10-60 IU/L]; aspartate aminotransferase [AST], $112 \mathrm{IU} / \mathrm{L}$ [normal range, 10-42 IU/L]), and all the other blood results revealed normal results.

Mrs HM was clinically stable on January 12, 2015, and all her treatment was continued. Ultrasound examination on January 14, 2015, indicated a disparity of 3 weeks between the gestational age and the findings on ultrasound examination: gestational age $35+4$ weeks of gestation by dates and ultrasound gestational age of $32+4$ weeks, there was a normal umbilical artery diastolic flow, a biophysical profile of $8 / 8$, an amniotic fluid index of 9.8 (normal), and an estimated fetal weight of $2.048 \mathrm{~kg}$. A diagnosis of fetal growth restriction (FGR)/intrauterine growth restriction was made. The nonstress test was now performed twice daily and a close monitoring of the mother was continued. The nonstress test demonstrated persistent fetal tachycardia: the mother was stable and had no pyrexia. The mother, however, demonstrated great anxiety because of the fetal tachycardia. Despite adequate counseling and reassurance, she insisted on immediate delivery. A decision was therefore taken to proceed with an emergency cesarean section which was successfully performed on January 14, 2015. A healthy, normal-looking female infant was delivered, with an Apgar score of 8 in 1 minute and 9 in 5 minutes and fetal birth weight of $2.290 \mathrm{~kg}$. The baby had mild respiratory distress and was immediately admitted in the neonatal intensive care unit and managed by the neonatologists. Mrs HM was treated routinely with intravenous antibiotics (ceftriaxone/ metronidazole). She had a superficial wound dehiscence, which was routinely treated, and her postoperative period was otherwise uneventful. Blood investigations revealed satisfactory hemoglobin and normal liver function tests. The baby spent 1 week in the neonatal intensive care unit under close observation, although no specialized treatment was required; the baby was then transferred to the Special Care Baby Unit for further surveillance and management. The baby was breast-fed by the mother. Mother and baby made satisfactory progress. The mother was discharged home with her baby on January 24, 2015.

\section{Discussion}

Mrs HM had been admitted with a diagnosis of RSM due to multiple etiological factors, which included hypothyroidism, PCOS, elevated NK cells, chromosome anomaly (balanced translocation), increasing maternal age, and obesity. The interaction/interconnection of these causes is complex. These factors and other etiological factors have been highlighted previously, ${ }^{1-3,9-12,14}$ and it is not unusual to have multiple etiological factors in the same patient. ${ }^{7,9,14}$ This patient was subjected to extensive investigations as previously outlined ${ }^{9,14}$ and these revealed the etiological factors. It was rather unfortunate that karyotype studies were delayed in this patient until the more recent review of her case at our clinic in January 2014, which revealed the additional etiological factor of balanced translocation. Mrs HM had a total of 13 previous miscarriages, and although evacuation was performed in ten of these miscarriages, the products of conception were not subjected to cytogenetic analysis, a practice that is not undertaken at our clinic, an investigation that may have revealed some forms of chromosomal abnormalities. In a recent study of cytogenetic analysis of products of conception 
in 43 patients with RSM with miscarriages, chromosome abnormalities were detected in $78 \%$ of the cases, ${ }^{30}$ and these authors concluded that in patients older than 35 years, fetal chromosome abnormalities were a major cause of the miscarriages. Our patient presented to our clinic at 37 years of age, and all her previous miscarriages were first-trimester miscarriages; however, the exact contribution of fetal chromosome malformations to these miscarriages could not be established because of the lack of appropriate investigations. Balanced translocation was diagnosed as a contributory cause of RSM in this patient, and PGD/ICSI/IVF was recommended for the couple because the husband of the patient had some problems in his semen analysis test. PGD/IVF has been recommended as a modality of treatment for balanced translocation, ${ }^{9,12}$ and this procedure with ICSI was offered to Mrs HM and resulted in the pregnancy that was managed with IVIG therapy and other modalities of medical therapy, which ultimately resulted in another successful pregnancy for this patient, the subject of the case report. The modalities of medical treatment referred earlier will be discussed later in this presentation.

A diagnosis of the etiological factors of PCOS and hypothyroidism had been made in the first successful pregnancy of Mrs HM managed in London, and these medical disorders were still under maintenance treatment. A treatment of the earlier medical problems in addition to elevated NK cells was managed with appropriate therapy and IVIG in that pregnancy which resulted in the first successful pregnancy and delivery of the patient, Mrs HM, in London in 2010. Investigations performed at the RSM clinic at maternity hospital in January 2014 confirmed the medical problems of PCOS and hypothyroidism and also revealed a diagnosis of elevated NK cells. Previous studies and reviews s, $^{1,16,17,19-23}$ had indicated that elevated NK cells were associated with an increased incidence of miscarriage and constitute an etiological factor for RSM. Some of these studies and others ${ }^{20,23,28,31}$ have reported that with appropriate treatment, the effect of the NK cells could be reduced/reversed and a high positive pregnancy outcome achieved. In the light of the earlier information, one can emphatically state that elevated NK cells play an important etiological role in RSM and have been included in the multiple etiological factors in the case reported. The mechanism through which elevated NK cells contribute to increased miscarriages and RSM include interference with the implantation process and alteration in the cytokine population in the uterus..$^{20,23,31}$ The treatment of elevated NK cells will be discussed later.

Pregnancies in PCOS patients may be associated with luteal phase deficiency, and this was anticipated in this patient and progesterone was prescribed as part of her medical treatment. Metformin had already been administered to this patient as a part of her treatment for PCOS before her pregnancy, and this treatment was continued all through the first trimester of the pregnancy. Metformin therapy had been associated with the reduction in the incidence of miscarriages and RSM and fetal loss. ${ }^{32}$ In a previous study, ${ }^{32}$ metformin therapy was associated with a significant reduction in the rate of spontaneous miscarriage (62\% to $26 \%$ ). No significant adverse effects or congenital malformations had been associated with metformin therapy in pregnancy, and metformin therapy has been successfully used for the management of diabetes mellitus in pregnancy.

A successful pregnancy has been associated with appropriate adjustment of the fetus to the immunological system of the mother, and this complex event in the maternal/fetal interface will not be discussed in this report. A successful pregnancy has also been associated with a shift from the T-helper (Th)1 cytokines to the T-helper (Th)2 cytokines; Th1 cytokines are regarded as cytotoxic and increase the incidence of miscarriages, whereas Th2 cytokines create an environment that enables successful pregnancy., ${ }^{9,33}$ Progesterone has been long established as playing a significant role in the successful establishment of pregnancy, and apart from its other endocrine functions in pregnancy, it has been known to play an immunomodulatory role in pregnancy. Dydrogesterone (Duphaston), an orally active progestogen, with many functions similar to pure endogenous progesterone, which has superior functions when compared with other progestogens, has been demonstrated in many studies to promote a shift in the Th1/Th2 cytokine ratio in favor of Th2 and thus promotes the continuation of a successful pregnancy. ${ }^{34}$ This information, among others, has formed the basis of the use of dydrogesterone in RSM patients in our clinic. Dydrogesterone had been ordered as a part of the treatment for Mrs HM in her first successful pregnancy in 2010 and was again prescribed for her in the index pregnancy. A recent study, ${ }^{35} \mathrm{a}$ double-blind, randomized, parallel, placebo-controlled trial, has presented a strong evidence for the use of dydrogesterone in patients with RSM; an improved pregnancy outcome and reduction in abortions were reported in the study: the risk of another miscarriage after three consecutive miscarriages was 2.4 times higher in the placebo group compared with that of the treatment group (risk ratio $=2.4 ; 95 \%$ confidence interval $[\mathrm{CI}]=1.3-5.9 ; P<0.001)$. In the study, dydrogesterone was continued until 20 weeks of gestation, although in our patient it was continued until 24 weeks of gestation, the standard practice in our RSM clinic. There were other positive 
benefits reported in the patients treated with dydrogesterone. $17 \alpha$-Hydroxyprogesterone caproate (primolut depot) was also prescribed for our patient to prevent another preterm labor because of her history of previous preterm delivery. This is a standard policy in our RSM clinic based on the recommendations of ACOG and supported by multiple previous studies and reviews on the benefits and use of progesterone preparations in the prevention of preterm labor.

The use of IVIG therapy in RSM has remained controversial and has produced a variety of results; indications for therapy have been varied, and the dosage of the drug, the time of commencement of the therapy, and the duration of therapy have been quite varied in the different studies

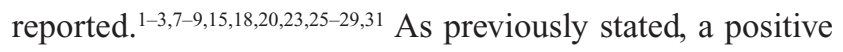
pregnancy outcome was reported in idiopathic secondary RSM, whereas there was no benefit for primary RSM. ${ }^{7}$ Positive pregnancy outcome was also reported in patients with secondary RSM; ${ }^{1,8,25-27,29}$ in some of these studies, the positive benefits were reported after secondary analyses of the available data. Positive pregnancy outcome was reported for secondary RSM in a meta-analysis of eight randomized controlled trials, ${ }^{29}$ whereas no benefit was reported for primary RSM. Another meta-analysis of six randomized trials found no benefit for both primary and secondary RSM. ${ }^{3}$ There was also no benefit reported for the use of IVIG in secondary idiopathic RSM in two recent randomized, double-blind, placebo-controlled trials; ${ }^{1,18}$ dosage regime of IVIG and duration of treatment in both trials were different $(0.5 \mathrm{~g} / \mathrm{kg}$ vs staggered dose of $0.4 \mathrm{~g} / \mathrm{kg}$ ). The use of IVIG therapy in patients with RSM due to elevated NK cells has been very impressive and consistent in some reports and produced highly positive results: pregnancy rates of $82 \%-96 \%$ have been reported and rapid decline/reduction of percentage NK cells was noted in the patients treated. ${ }^{20,23,28,31}$ The dosage of IVIG used in these studies was also different: $0.5 \mathrm{~g} / \mathrm{kg}$ in one study ${ }^{23}$ and $0.4 \mathrm{~g} / \mathrm{kg}$ in other studies, ${ }^{20,31}$ and the time of commencement of IVIG therapy and duration of treatment (15-35/36 weeks) varied. In our patient, IVIG therapy was administered because of elevated NK cells in both successful pregnancies, and the dosage regime $(0.5 \mathrm{~g} / \mathrm{kg})$ and duration of treatment were identical. In our patient, Mrs HM, 13 previous pregnancies ended in first-trimester miscarriages. There was no evidence of any specific therapy or the use of IVIG in any of these failed pregnancies. In her first successful pregnancy managed in London in 2010, she was treated with supportive medical treatment and IVIG targeting the various etiological factors detected; IVIG therapy was continued till 30 weeks of gestation, a gestational age that had been selected because it was felt that a more favorable gestational age for fetal survival had been achieved and this could be further enhanced with neonatal services (should early delivery be recorded). This was the basis of the utilization of similar medical treatment and IVIG therapy. IVIG therapy was continued to 30 weeks of gestation in the second pregnancy, the index pregnancy of the case report based on the evidence of the previous successful pregnancy. The evidence of positive outcome of previous pregnancies using metformin, dydrogesterone, and IVIG has been provided in the reports previously cited in this report. One can therefore deduce that the two successful pregnancies recorded in this patient were due to the medical treatment and IVIG therapy targeting the multiple etiological factors causing RSM in the patient. Thus, one can state that in our patient IVIG was $100 \%$ successful in the treatment of her elevated NK cells and other etiological factors. The contribution of PGD/ICSI/IVF to the current successful pregnancy must be recognized as a positive intervention as these procedures had been associated with successful pregnancies in previous reviews.

The gestational age at delivery was significantly higher in the patients treated with dydrogesterone ${ }^{35}$ and in the patients with secondary idiopathic $\mathrm{RSM}^{18}$ treated with IVIG compared with that of the controls; there was also a tendency toward the reduction of prematurity, and the fetal birth weights were generally satisfactory. These positive benefits were not observed in the consecutive successful pregnancies reported in our patient since both pregnancies ended at 36 weeks of gestation, and in the second pregnancy, FGR/intrauterine growth restriction was actually diagnosed. Other complications recorded in the second pregnancy, subject of the case report, APH, and cholestasis of pregnancy, are not peculiar and linked to the modalities of treatment offered to our patient, and we want to observe that FGR has also been reported in some of the pregnancies of patients treated with IVIG. ${ }^{29}$ The successful pregnancy in this patient was most likely a result of the interaction and interconnection of all the forms of medical therapy and IVIG, the interventions targeting the multiple etiological causes of RSM in this patient. On the whole, the course of the pregnancy in our case report was satisfactory, and the various modalities of treatment offered to the patient did not cause any adverse effects.

Although the exact mechanism of the action of IVIG in the treatment of RSM has not been well established, ${ }^{3}$ various suggestions have been made to explain the mechanism of action. These include an immunomodulatory role, modulation of cytokines/production of helper suppressor T-cell 
cytokines, downregulation of systemic NK cell activity, and reduction of NK cell activity at the implantation site thus reducing the likelihood of miscarriages and anti-infective/ anti-inflammatory activity. 3,9,15,36

The unresolved problems of the use of IVIG in RSM include the following: the indication for IVIG, the dosage regime, gestational age at the commencement of therapy, the duration of therapy, and the gestational age at the termination of the therapy. These problems need to be resolved and standardized to facilitate the comparison of trials on the use of IVIG therapy in RSM from different centers. Our case report has indicated a modality of treatment with IVIG therapy ending at 30 weeks of gestation, which needs to be replicated in further studies. The problems highlighted earlier call for larger, double-blind, randomized, placebo-controlled (multicentered) trials before a definitive conclusion can be made on the role, treatment regimes, and usefulness of IVIG in the management of RSM.

Although IVIG is quite expensive and this may limit the use of IVIG in RSM in many centers as well as having an effect on the size of planned trials, this high cost is not a limitation for the use of IVIG in selected patients with RSM in our clinic because such therapy is fully financed by the government. IVIG therapy has been associated with some minor side effects although few major side effects have been reported.

\section{Conclusion}

We have presented Mrs HM, 37 years old, $\mathrm{P} 0+1+13+1$, with high-order RSM due to multiple etiological factors, including balanced translocation, PCOS, hypothyroidism, elevated NK cells, moderate obesity, and advanced age of older than 35 years, with two consecutive successful pregnancies (and PGD/ICSI/IVF in the latter pregnancy) and multiple supportive medical therapy and IVIG therapy targeting the etiological factors. This report has demonstrated that IVIG may have a positive role in the management of RSM. We call for further, well-planned, larger, double-blind, randomized, placebo-controlled trials to resolve the problems of the real benefits of the use of IVIG therapy in RSM and the many unresolved problems highlighted earlier which are associated with IVIG therapy.

\section{Acknowledgments}

We are grateful to all our colleagues for their contribution to the successful management of our patient. We are very grateful to Mrs Anju Nair for her technical support in the preparation of the manuscript.

\section{Disclosure}

The authors report no conflicts of interest in this work.

\section{References}

1. Stephenson MD, Kutteh WH, Purkiss S, et al. Intravenous immunoglobulin and idiopathic secondary recurrent miscarriage: a multicentered randomized placebo-controlled trial. Hum Reprod. 2010;25: 2203-2209.

2. Daya S, Gunby J, Porter F, Scott J, Clark DA. Critical analysis of intravenous immunoglobulin therapy for recurrent miscarriage. Hum Reprod Update. 1999;5:475-482.

3. Ata B, Tan SL, Shehata F, Hozler H, Buckett W. A systematic review of intravenous immunoglobulin for treatment of unexplained recurrent miscarriage. Fertil Steril. 2011;95:1080-1085.

4. Reagan L. A prospective study of spontaneous abortion. In: Beard RW, Sharp F, editors. Early Pregnancy Loss: Mechanisms of Treatment. London: Springer-Verlag; 1988:23-27.

5. Wilcox AJ, Weinberg CR, O'Connor JF, et al. Incidence of early loss of pregnancy. N Engl J Med. 1988;319:189-194.

6. Alberman E. The epidemiology of repeated abortions. In: Beard RW, Sharp F, editors. Early Pregnancy Loss: Mechanisms of Treatment. London: Springer-Verlag; 1988:9-17.

7. Christiansen OB, Pederson B, Rosgaard A, Husth M. A randomized; double-blind, placebo-controlled trial of intravenous immunoglobulin in the prevention of recurrent miscarriage: evidence of a therapeutic effect in women with secondary recurrent miscarriage. Hum Reprod. 2002;17:809-816.

8. Rai R, Reagan L. Recurrent miscarriage. Lancet. 2006;368: 601-611.

9. Diejomaoh MF. Recurrent spontaneous miscarriage is still a challenging, diagnostic and therapeutic quagmire. Med Princ Pract. 2015;24 (Suppl I):38-55.

10. The practice committee of the American Society for Reproductive medicine. Evaluation and treatment of recurrent pregnancy loss. A committee opinion. Fertil Steril. 2012;98:1103-1111.

11. Jaslow CR, Carney JL, Kutteh WH. Diagnostic factors identified in 1020 women with two versus three or more recurrent pregnancy losses. Fertil Steril. 2010;93:1234-1243.

12. Brezina PR, Kutteh WH. Classic and cutting-edge strategies for the management of early pregnancy loss. Obst Gynecol Clin North Am. 2014;4:1-18.

13. Shapira E, Ratzon R, Shoham-Vardi I, Serjienko R, Mazor M, Bashiri A. Primary versus secondary recurrent pregnancy loss-epidemiological characteristics, etiology, and next pregnancy outcome. $J$ Perinat Med. 2012;40:389-396.

14. Diejomaoh MF, Al-Azemi M, Jirous J, et al. The aetiology and pattern of recurrent pregnancy loss. J Obstet Gynaecol. 2002; 22:62-67.

15. Porter TF, La Coursiere Y, Scott JR. Immunotherapy for recurrent miscarriage. Cochrane Database Syst Rev. 2006;2:CD000112.

16. Quenby S, Bates M, Doig T, et al. Pre-implantation endometrial leukocytes in women with recurrent miscarriage. Hum Reprod. 1999;14: 2386-2391.

17. King K, Smith S, Chapman M, Sacks G. Detailed analysis of peripheral blood natural killer [NK] cells in women with recurrent miscarriage. Hum Reprod. 2010;25:52-58.

18. Christiansen OB, Larsen EC, Egerup P, Lunoee L, Egestad L, Nielsen HS. Intravenous immunoglobulin treatment for secondary recurrent miscarriage: a randomised, double-blind, placebo-controlled trial. BJOG. 2015;122:500-508.

19. Clifford K, Flanagan AM, Reagan L. Endometrial CD56+ natural killer cells in women with recurrent miscarriage: a histomorphometric study. Hum Reprod. 1999;14:2727-2730.

20. Ramos-Medina R, García-Segovia A, Gil J, et al. Experience in IVIg therapy for selected women with recurrent reproductive failure and NK cell expansion. Am J Reprod Immunol. 2014;71:458-466. 
21. Aoki K, Kajiura S, Matsumoto Y, et al. Preconceptional natural killer - cell activity as a predictor of miscarriage. Lancet. 1995;345: 1340-1342.

22. Yamada H, Morikawa M, Kato EH, Shimada S, Kobashi G, Minakami H. Pre-conceptional natural killer cell activity and percentage as predictors of biochemical pregnancy and spontaneous abortion with normal chromosome karyotype. Am J Reprod Immuol. 2003;50:351-354.

23. Perricone R, Di Muzio G, Perricone C, et al. High levels of peripheral blood NK cells in women suffering from recurrent spontaneous abortion are reverted from high-dose intravenous immunoglobulin. Am J Reprod Immunol. 2006;55:232-239.

24. Silver RM. Intravenous immune globulin: another disappointing treatment for pregnancy loss. BJOG. 2015;122:509.

25. Christiansen OB, Mathiesen O, Husth M, et al. Placebo-controlled trial of treatment of unexplained secondary recurrent spontaneous abortions with IV. Immunoglobulin. Hum Reprod. 1995;19:2690-2695.

26. Stephenson MD, Dreher K, Honlihan E, Wu V. Prevention of unexplained recurrent spontaneous abortion using intravenous immunoglobulin: a prospective, randomized, double blinded, placebo-controlled trial. Am J Reprod Immunol. 1998;39:82-88.

27. Jablonowska B, Selbing A, Palfi M, Ernerudh J, Kjellberg S, Lindton B. Prevention of recurrent spontaneous abortion by intravenous immunoglobulin: a double-blind placebo-controlled study. Hum Reprod. 1999; $14: 838-841$.

28. Coulam CB, Acacio B. Does immunotherapy for treatment of reproductive failure enhance live births? Am J Reprod Immunol. 2012;67: 296-303.
29. Hutton B, Sharma R, Fergusson D, et al. Use of intravenous immunoglobulin for recurrent spontaneous miscarriage: a systemic review. BJOG. 2007;114:134-142.

30. Marquard K, Westphal LM, Milki AA, Lathi RB. Etiology of recurrent pregnancy loss in women over the age of 35 years. Fertil Steril. 2010;94: 1473-1477.

31. Moraru M, Carbone J, Alecsandru D, et al. Intravenous immunoglobulin treatment increased live birth rate in a Spanish cohort of women with recurrent reproductive failure and expended CD 56+ cells. Am J Reprod Immunol. 2012;68:75-84.

32. Glueck CJ, Wang P, Goldenberg N, Sieve-Smith L. Pregnancy outcomes among women with polycystic ovary syndrome treated with metformin. Hum Reprod. 2002;17:2858-2864.

33. Szekeres-Bartho J. Immunologic relationship between the mother and fetus. Intern Rev Immunol. 2002;21:471-496.

34. Raghupathy R, Al Mutawa E, Makhseed M, Azizieh F, Szekeres-Bartho J. Modulation of cytokine production by dydrogesterone in lymphocytes from women with recurrent miscarriage. BJOG. 2005;112: 1096-1101.

35. Kumar A, Begum N, Prasad S, Aggarwal S, Sharma S. Oral dydrogesterone treatment during early pregnancy to prevent recurrent pregnancy loss and its role in modulation of cytokine production: a double-blind, randomized, parallel, placebo-controlled trial. Fertil Steril. 2014;102:1357-1363.

36. Leong H, Stachnik J, Bonk ME, Matuszewski MS. Unlabeled uses of intravenous immunoglobulin. Am J Health Syst Pharm. 2008;65: $1815-1824$
International Medical Case Reports Journal

\section{Publish your work in this journal}

The International Medical Case Reports Journal is an international, peer-reviewed open-access journal publishing original case reports from all medical specialties. Previously unpublished medical posters are also accepted relating to any area of clinical or preclinical science. Submissions should not normally exceed 2,000 words or

\section{Dovepress}

4 published pages including figures, diagrams and references. The manuscript management system is completely online and includes a very quick and fair peer-review system, which is all easy to use. Visit http://www.dovepress.com/testimonials.php to read real quotes from published authors. 